\title{
Radiation Sensitivity of in Vitro Evaluation System of Pharmacokinetics in Boron Neutron Capture Therapy (BNCT) Using Three-Dimensional Artificial Human Tumor Tissue Model
}

\author{
Shintaro Ishiyama1 ${ }^{*}$, Minoru Suzuki ${ }^{2}$ \\ ${ }^{1}$ Faculty of Science and Technology, Graduate School of Science and Technology, Hirosaki University, Bunkyo, Hirosaki, \\ Aomori, Japan \\ ${ }^{2}$ Particle Radiation Oncology Research Center, Institute for Integrated Radiation and Nuclear Science, Kyoto University, \\ Asahiro-nishi, Kumamori, Sennan, Osaka, Japan \\ Email: ${ }^{*}$ ishiyama.shintaro@hirosaki-u.ac.jp
}

How to cite this paper: Ishiyama, S. and Suzuki, M. (2019) Radiation Sensitivity of in Vitro Evaluation System of Pharmacokinetics in Boron Neutron Capture Therapy (BNCT) Using Three-Dimensional Artificial Human Tumor Tissue Model. Journal of Cancer Therapy, 10, 1025-1035. https://doi.org/10.4236/jct.2019.1012086

Received: November 9, 2019

Accepted: December 27, 2019

Published: December 30, 2019

Copyright $\odot 2019$ by author(s) and Scientific Research Publishing Inc. This work is licensed under the Creative Commons Attribution International License (CC BY 4.0).

http://creativecommons.org/licenses/by/4.0/

\begin{abstract}
One of the important matters that must be determined in advance when performing BNCT treatment is the optimization of neutron irradiation time and dose. In this article, following the previous article $\left(2.52 \times 10^{12} \mathrm{n} / \mathrm{cm}^{2}\right)($ Case 1 ), double irradiation $\left(5.04 \times 10^{12} \mathrm{n} / \mathrm{cm}^{2}\right)$ was further performed (Case 2) by verifying the radiation sensitivity performance of the artificial tumor tissue NHDF3D/BxPC3 and the possibility of evaluating the optimum neutron dose required for treatment was examined. As a result, although the radiation damage rate in the normal tissue NHDF3D and the tumor tissue BxPC3 increased in proportion to the irradiation dose due to heavy irradiation in Case 1 or more, the increase in the damage rate in the normal tissue exceeded the tumor tissue. Furthermore, the tumor/normal tissue damage ratio $\mathrm{T} / \mathrm{N}$ ratio showed the maximum value in Case 1, and the dose ratio in Case 2 with a higher dose showed a tendency to decrease. From the above experimental facts, it was shown that irradiation dose optimization is possible to some extent by an evaluation method using an artificial tumor tissue.
\end{abstract}

\section{Keywords}

Boron Neutron Capture Therapy (BNCT), Boronophenylalanine $\left({ }^{10} \mathrm{BPA}\right)$, Artificial Human Tumor Tissue Model, Cell Accumulation Method 


\section{Introduction}

In the previous paper [1], the authors made a bilayer 3D artificial tumor tissue (BxPC3/NHDF3D) using human pancreatic cancer cell line BxPC3 and normal human dermal-derived fibroblast NHDF3D, and pharmacokinetic study for 10BPA-BNCT treatment [2]-[10] by neutron irradiation from reactor Went. As a result, optical observation of the irradiated tissue gave a $\mathrm{T} / \mathrm{N}$ ratio of 3.19 at a neutron dose of $2.52 \times 10^{12} \mathrm{n} / \mathrm{cm}^{2}$, indicating the effectiveness of the BNCT pharmacokinetics test using 3D artificial tissue [11]-[16].

On the other hand, what is important in BNCT treatment of tumor patients is to secure the accumulation in the tumor affected area after the boron drug administration and to optimize the irradiation dose during the treatment.

Therefore, in this paper, we examined the applicability of the 3D artificial tumor tissue used in the previous paper [1] as a testing technique for optimizing the radiation dose at the time of BNCT treatment while verifying the sensitivity performance to radiation dose.

\section{Materials and Methods}

\subsection{Cells, Reagents and Instruments}

Normal human dermal-derived fibroblast (NHDFs) and red fluorescent protein (RFP) -labeled human pancreatic cancer cell line BxPC3 used in the experiment were purchased from LONZA (Walkersville, MD) and Anti-Cancer Japan (Ibaraki, Japan), respectively. Dulbecco's modified Eagle's medium (DMEM) (Wako, Osaka, Japan) containing 10\% fetal bovine serum (FBS) (Nichirei, Tokyo, Japan) was used to proliferate cells prior to construction of the tumor tissue model. The cells were cultivated at $37^{\circ} \mathrm{C}, 5 \%$ carbon dioxide. Bovine plasma-derived fibronectin (FN) and porcine skin gelatin (G) were purchased from Sigma-Aldrich (St. Louis, MO) and Wako Pure Chemical Industries, Ltd. (Osaka, Japan), respectively. Transwell inserts with porous polyester bottom (pore size: $0.4 \mu \mathrm{m}$ ) for 12-well culture plate (12 mm diameter, $112 \mathrm{~mm}^{2}$ area, cat. No. 3401) were purchased from CORNING Inc. (New York, NY). The boron drug ${ }^{10} \mathrm{BPA}$ (boronophenylalanine; $\mathrm{C}_{9} \mathrm{H}_{12} \mathrm{BNO}_{4}$, molecular weight 209.01, fructose complex) was kindly gifted from Interpharma Praha, a.s. (Komorany, Czechia). A solid-state nuclear track detector CR-39, an optical plastic material with composition $\mathrm{C}_{12} \mathrm{H}_{18} \mathrm{O}_{7}$ [7] [8], was purchased from Cokin (Tokyo, Japan) [1].

\subsection{Preparation of in Vitro Human Three-Dimensional Tumor Tissue Model}

Human three-dimensional tumor tissue was prepared by cell accumulation method [11] [12] [13] [14]. First, connective tissue-like structures were fabricated by three-dimensional lamination of NHDFs. As shown in Figure 1, NHDFs are cultured under the conditions mentioned above, then ECM-nano film (about 10 $\mathrm{nm}$ thick) was formed on each cell surface by coating cells with fibronectin and gelatin dissolved in Tris- $\mathrm{HCl}$ buffer according to previously published methods 
(a)

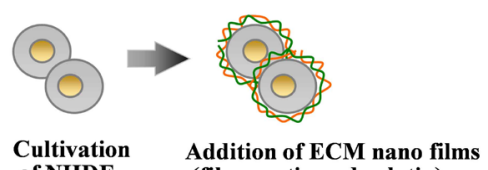
of NHDF Addition of ECM nano film
(fibronectin and gelatin)
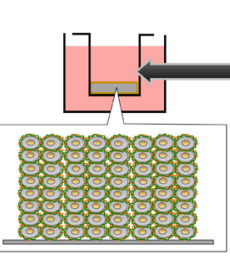

3D-caltivation of coated NHDF (eight layers)

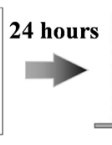

urs Tissue construction (normal tissue model = NHDF3D)

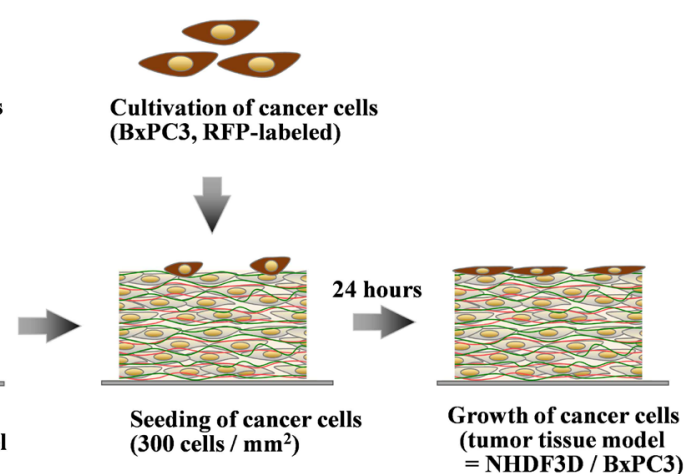

(b)

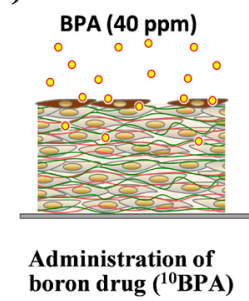

(c)

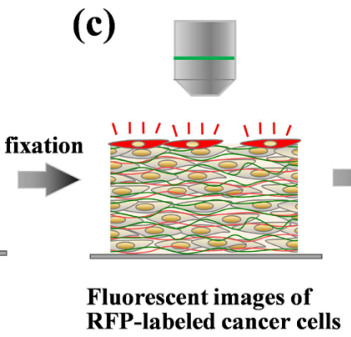

(d)

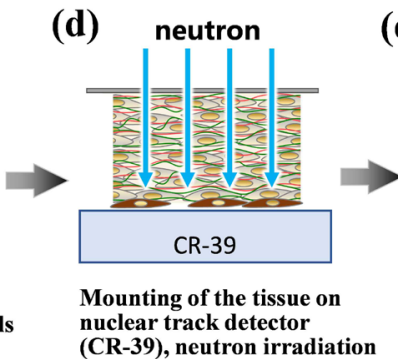

(e) NHDF3D / BxPC3

Figure 1. Procedures for detection of BNCT reaction by using in vitro three-dimensional artificial human tumor tissue model. (a) Fabrication process of cancer cells-loaded human artificial tissue model. (b)-(e) Operation of BNCT for the tissue models, and detection of cancer cell and nuclear track distribution. In present paper, we adopted combination of an artificial tumor tissue model, comprised of normal human dermal-derived fibroblast (NHDF) and human pancreatic cancer cell line BxPC3.

[11] [12] [13] [14]. The cells are seeded on the transwell inserts at a density of $27.2 \times 10^{5}$ cells/insert (8 layers) and cultured under the conditions of $5 \%$ carbon dioxide at $37^{\circ} \mathrm{C}$ for 12 to 24 hours. We regarded this connective tissue-like structure as an artificial human normal tissue model, termed as NHDF3D [1].

Next, RFP-labeled BxPC3 cells which have been cultured and proliferated were collected by trypsin treatment, washed, and uniformly seeded on the upper surface of NHDF3D at a density of 300 cells $/ \mathrm{mm}^{2}$, then further cultured for 24 hours under the above culture conditions. BxPC3 cells proliferated on the surface of NHDF3D forming the groups with a flat shape. We regarded this cancer-loaded NHDF3D as an artificial human tumor tissue model, termed as NHDF3D/BxPC3. In the present study, one case of NHDF3D and three cases of $\mathrm{NHDF} 3 \mathrm{D} / \mathrm{BxPC} 3$ were prepared by the methods mentioned above and were used for the experiments.

\subsection{BPA Immersion Treatment and Fixation}

The boronophenylalanine (BPA) solution $(3 \% \mathrm{w} / \mathrm{v})$ was diluted to a concentration of $40 \mathrm{ppm}$ with DMEM containing $10 \%$ FBS. This is referred to as a BPA treatment solution. After removing the culture solution of NHDF3D or NHDF3D/BxPC3, $750 \mu$ of BPA treatment solution was added, and incubated 
for 2 hours (BPA exposure) under conditions of $5 \%$ carbon dioxide at $37^{\circ} \mathrm{C}$. After that, the BPA treatment solution was removed and the tissues were washed 3 times with $0.01 \mathrm{M}$ phosphate buffered saline (PBS, pH 7.3). Subsequently, the tissues were fixed by $4 \%$ paraformaldehyde/0.1 M phosphate buffer ( $\mathrm{pH}$ 7.3) for 30 minutes at room temperature shading the light. After the fixation, the cellular nucleus was stained by 4',6-diamidino-2-phenylindole (DAPI) [1].

\subsection{Observation of BxPC3 cell Distribution on the Artificial Tissues}

After the fixation, three holes were provided on the tissue using an $18 \mathrm{G}$ injection needle in order to provide alignment marks. Then the top surface of NHDF3D/BxPC3 was observed by fluorescence microscope BZ-X700 (Keyence, Osaka, Japan). The distribution of BxPC3 cells was visualized as a fluorescence image by RFP excitation. A low magnification image including the entire tissue and high magnification images of various parts of each tissue were respectively obtained. A fluorescence image of NHDF3D was also obtained as a control [1].

\subsection{Neutron Irradiation Experiment}

The above-mentioned NHDF3D or NHDF3D/BxPC3 in the transwell inserts were cut out with a knife together with the polyester base, mounted on the solid track detector CR-39 [7] [8] with close contact, and used as a sample for track image acquisition (Figure 1(d)). Irradiation experiments using these samples were conducted at the Heavy Water Neutron Irradiation Facility of Kyoto University Reactor (KUR), and irradiation was performed for 60 minutes under an irradiation flux of $1.4 \times 10^{9} \mathrm{n} / \mathrm{cm}^{2} / \mathrm{s}$ (total flux $=4.5 \times 10^{12} \mathrm{n} / \mathrm{cm}^{2}$ ). After the neutron irradiation, the above sample was etched $\left(6 \mathrm{~N} \mathrm{NaOH}, 70^{\circ} \mathrm{C} \times 2\right.$ hours) to visualize the $\alpha$-ray/recoiled Li particle tracks generated on the CR-39 surface [1].

\subsection{Analysis of $\alpha$-Ray/Recoiled Li Particle Track Images}

The $\alpha$-ray/recoiled Li particle track image of etched CR-39 was taken using the bright field function of the fluorescence microscope BZ-X700. A low magnification image including the entire tissue mount and 10 random high magnification images were obtained respectively, and the following analysis was performed.

1) The whole tissue images of fluorescent $\mathrm{BxPC} 3$ cell distribution and the $\alpha$-ray/ recoiled Li particle track distribution were compared referring to the position of three-hole markers, and the relationship of these distributions was observed.

2) Alpha-ray/recoiled Li particle tracks in the high magnification images were regarded as the particles, and quantitatively analyzed by using software FIJI (https://fiji.sc). Briefly, after binarizing the image, the tracks with more than 4 $\mu \mathrm{m}$ diameter were detected, and their number per unit area $\left(0.01 \mathrm{~mm}^{2}\right)$ and the track size were measured. A significant difference between the data from the artificial normal tissue model (NHDF3D) and artificial tumor tissue model (NHDF3D/BxCP3) was confirmed using student $t$ test [1]. 


\section{Results and Discussions}

\subsection{Distribution of the BXPC3 Cells and Alpha-Ray/Recoiled Li Particle Tracks on the Tissue Models}

Low-magnification images of NHDF3D and NHDF3D/BxPC3 (three cases) detecting RFP fluorescence were shown in Figure 2(a) (NHDF3D) and Figures 2(b)-(d) (NHDF3D/BxPC3) before irradiation. Although the BxPC3 cells (Figures 2(b)-(d)) were seeded over the artificial tissue (Figure 2(a)) in regular cell concentration, slight heterogeneity of cell distribution was observed after the cultivation for 24 hours.

The $\alpha$-ray/recoiled Li particle tracks in corresponded parts of Figures 2(a)-(d) were shown in Figures 3(a)-(d). The marked \#1 - \#12 in the figure are measurement points that have been observed by high-magnification optical observation in Figure 3.

Figure 4 group (a) (3DNHDF) and group (b) (NHDF3D/BxPC3), and Figure 4 group (c) (d) (NHDF3D/BxPC3) showed the distribution of $\alpha$-ray/recoiled Li particle tracks in 3DNHDF and NHDF3D/BxPC3, respectively.

In these figures, the $\alpha$-ray/recoiled Li particle tracks were observed as small dots at \#1 - \#12, reflecting the intensity of the emission. These tracks distribution and density at different observation points in each tissue are approximated for each group. This means that the $\alpha$-ray/recoiled Li particle damage occurs almost uniformly in each tissue.

Since the contrast between NHDF3D (Figure 4(a)) and NHDF3D/BxPC3

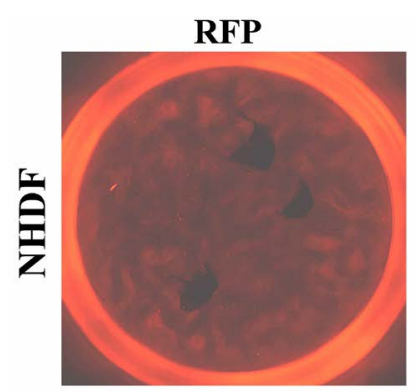

(a)

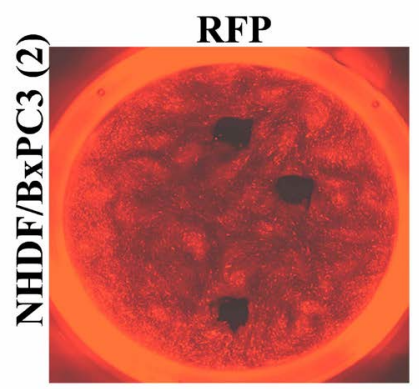

(c)

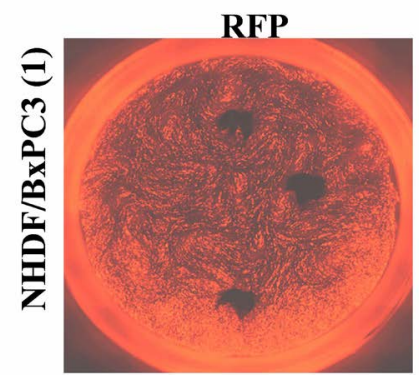

(b)

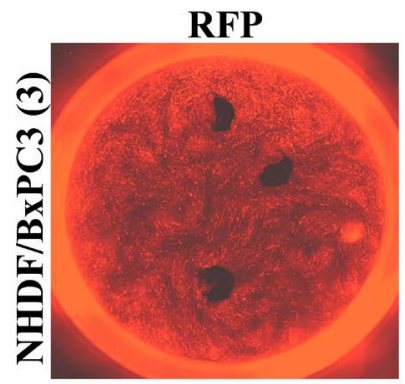

(d)

Figure 2. Comparision of Fluorescent staining of NHDF3D and BxPC3 cells. (a)-(d) Microscopic images of NHDF3D and BxPC3 cells on culture dish. (a) NHDF3D. (b)-(d) red fluorescence image of RFP-labeled BxPC3 cells. 
CR-39

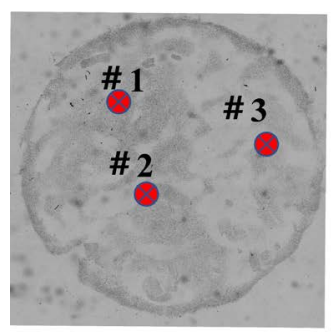

(a)

CR-39

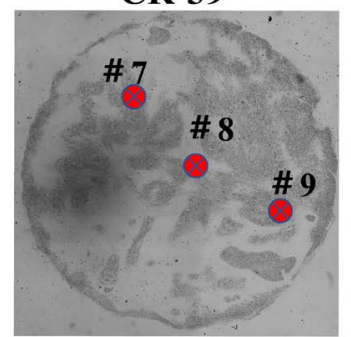

(c)
CR-39

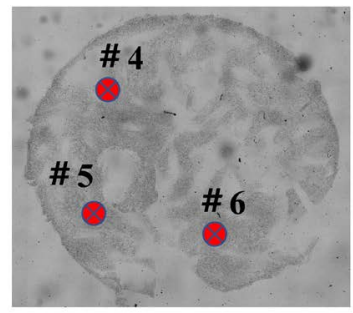

(b)

CR-39

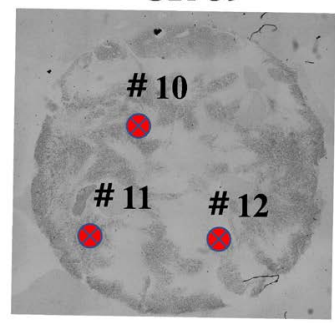

(d)

Figure 3. Comparison of $\alpha$-ray/recoiled Li particle track distribution in low magnification. (a) NHDF3D as a control without BxPC3 cells. (b)-(d) three cases of cancer-loaded tissue; NHDF3D/BxPC3 $(1-3)$. The marked \#1 to \#12 in the figure are measurement points that has been observed by high-magnification optical observation in Figure 4.

(Figure 4(b) and Figure 4(c) Figure 4(d)) was obvious, the BxPC3 cells-dependent incorporation of ${ }^{10} \mathrm{~B}$ resulting the $\alpha$-ray/recoiled Li particle emission was clearly detected in this system [1].

\subsection{Radiation Sensitivity of NHDF3D and NHDF3D/BxCP3}

Here, we verified the suitability as a tool to determine the dose optimization during BNCT treatment by using NHDF3D/BxPC3.

To investigate detailed $\alpha$-ray/recoiled Li particle track density in NHDF3D and NHDF3D/BxCP3 samples, high magnification images of $\alpha$-ray/recoiled Li particle tracks on CR-39 [Figure 5(A) Figure 5(B) and Figure 5(a') Figure $5\left(b^{\prime}\right)$, photo] were quantitatively analyzed. Here, Figure 5(a') Figure 5(b') correspond to Figure 4(a) and Figure 4(c), respectively. Figure 5(A) and Figure 5(B) show $\alpha$-ray/recoiled Li particle tracks observed in NHDF3D and NHDF3D/BxPC3, when irradiated with Case $1\left(2.52 \times 10^{12}\right)$ [1] and Figure 5(a') Figure 5(b') with Case $2\left(4.5 \times 10^{12} \mathrm{n} / \mathrm{cm}^{2}\right.$ neutron dose), respectively. Table 1 shows the results of measuring the density of $\alpha$-ray/recoiled Li particle tracks in the unit area $\left(0.01 \mathrm{~mm}^{2}\right)$ in Case 1 and Case 2.

There is a clear difference in track density between the two, and it can be seen that the higher the neutron irradiation amount, the higher the track density. Similarly, when the track density of NHDF3D/BxPC3 was compared between Figure 5(B) and Figure 5(b'), it was found that the track density was high at the high dose NHDF3D/BxPC3. 

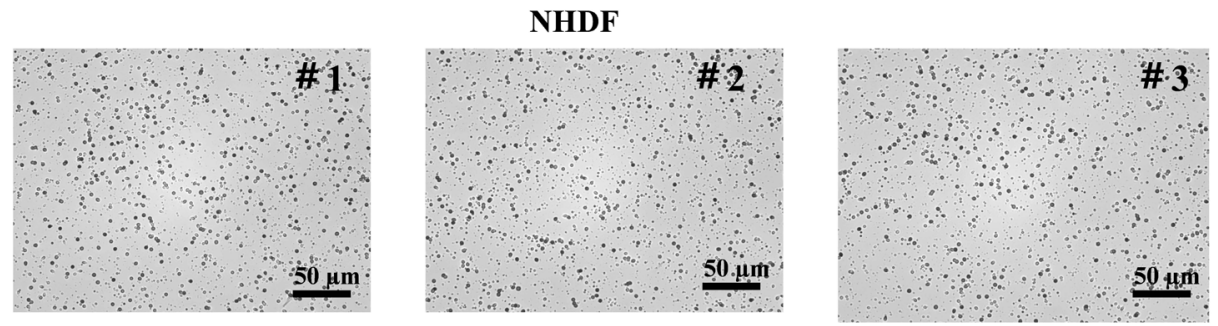

(a)

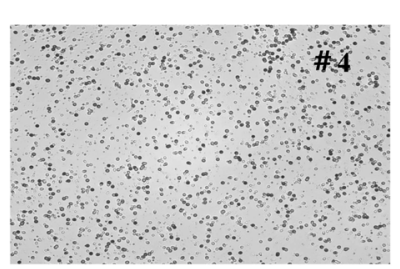

\section{NHDF/BxPC3 (1)}
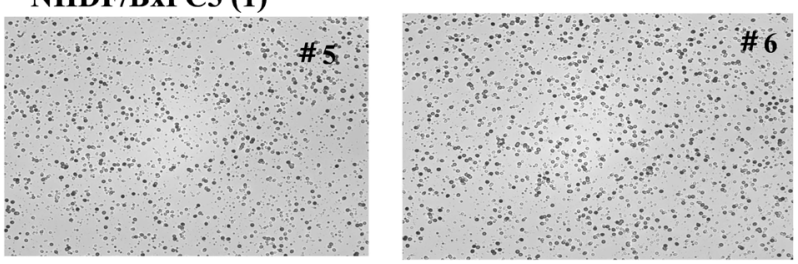

(b)
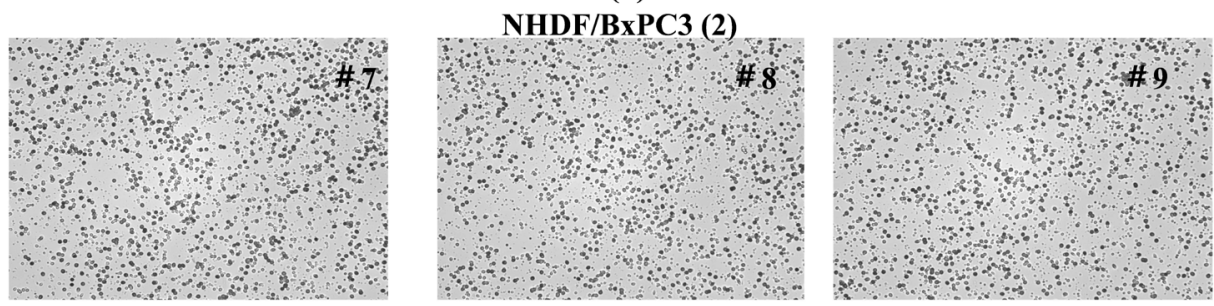

(c)

NHDF/BxPC3 (3)
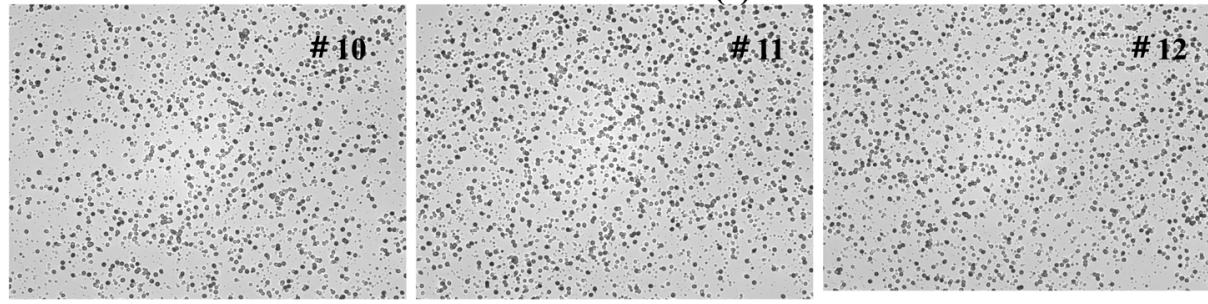

(d)

Figure 4. Comparison of NHDF3D and BxPC3 cell distribution and $\alpha$-ray/recoiled Li particle track distribution in higher magnification. Groupe (a) and (b): High-magnification optical observation $\alpha$-ray/recoiled Li particle track distribution at observation points \#1 - 6. Groupe (a): NHDF3D. Groupe (b): NHDF3D/BxPC3 (1); Groupe (c) (d): High-magnification optical observation $\alpha$-ray/recoiled Li particle track distribution at observation points \#7 - 12. Groupe (c) and Groupe (d): NHDF3D/BxPC3 (2 - 3).

Table 1. $\alpha$-ray/recoiled Li particle tracks density (counts $/ 0.01 \mathrm{~mm}^{2}$ ), and $\mathrm{T} / \mathrm{N}$ ratio of NHDF3D/BxPC3.

\begin{tabular}{cccc}
\hline Cells & Case 1 [1] & Case 2 & Case 2/Case 1 \\
\hline NHDF3D & 51.52 & 127.5 & 2.48 \\
BxPC3(1) & 81.10 & 146.2 & 1.80 \\
BxPC3(2) & 87.68 & 140.3 & 1.60 \\
BxPC3(3) & - & 137.9 & - \\
$T / N$ & 1.63 & 1.11 & - \\
\hline
\end{tabular}




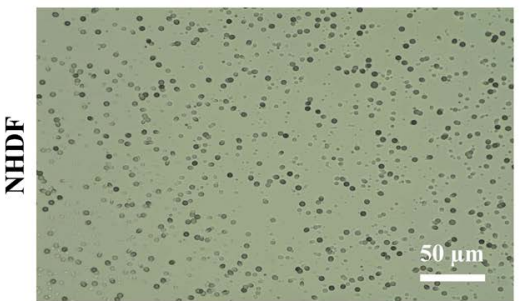

(A)

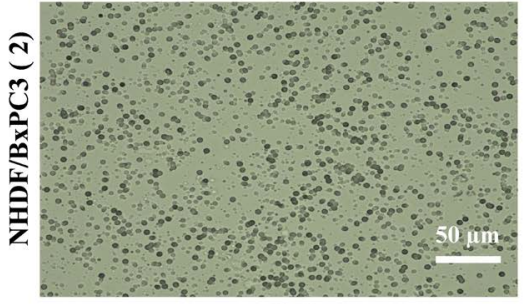

(B)

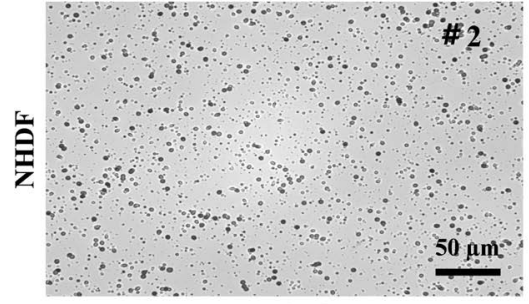

(a')

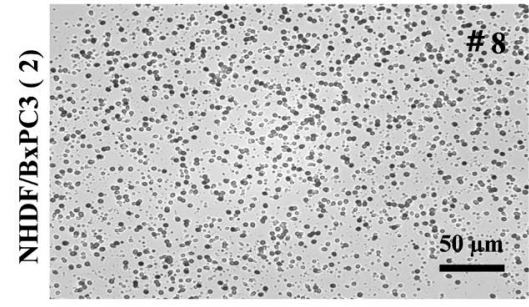

(b')

Figure 5. Comparison of $\alpha$-ray/recoiled Li particle tracks distribution in NHDF3D and NHDF3D/BxCP3 samples. High magnification photo images of $\alpha$-ray/recoiled Li particle track distribution were processed by using software Fiji for quantitative analysis of $\alpha$-ray/recoiled Li particle tracks. (A): NHDF3D of Case 1 and (a'): NHDF3D of Case 2. (B): NHDF3D/BxPC3 of Case 1 and (b') NHDF3D/BxPC3 (2) of Case 2. The binary data were prepared from the photo images and particle analysis was performed to count the number of the tracks and measure the of their size and the number of the particle tracks in $0.01 \mathrm{~mm}^{2}$ (track density). By further expanding the tissue change sites in the normal tissue and tumor tissue in the artificial tumor tissue after BNCT treatment, it was possible to grasp in detail the $\alpha$ track shape and distribution that occurred after BNCT treatment in Case 1 and Case 2.

The track density increases as the irradiation dose increases in this way, meaning that ${ }^{10} \mathrm{~B}$ absorbed in the sample remains in the tissue in an unreacted state even after neutron irradiation for a short time of about 30 minutes [1].

Figure 6 shows the relationship between neutron irradiation dose $\left(\mathrm{n} / \mathrm{cm}^{2}\right)$ and track density (counts $/ 0.01 \mathrm{~mm}^{2}$ ). According to this, the increase rate of NHDF3D and $\mathrm{BxPC} 3$ is different with the increase of the irradiation amount, and the increase rate of the track density of NHDF3D is remarkable. On the other hand, it can be seen that the increase rate of $\mathrm{BxPC} 3$ is almost saturated, however, in Case 2 , the rate of increase is similar to that of normal cells.

This means that when neutrons dose reached up to $4.25 \times 10^{12} \mathrm{n} / \mathrm{cm}^{2}$ or more, the effect of radiation damage on normal cells must be considered in addition to the original BNCT treatment effect.

Figure 7 shows the relationship between the neutron irradiation dose and the $T / N$ ratio, where the definition of $T / N$ ratio here is defined as the track density ratio of NHDF3D and BxPC3 in Table 1. According to this, although the $T / N$ ratio increases as the neutron irradiation dose increases, it turns to decrease when the irradiation dose in Case 1 is exceeded. In other words, in Case 2 from the viewpoint of BNCT treatment, radiation damage to the normal tissue becomes significant and there is a possibility of over-irradiation. 


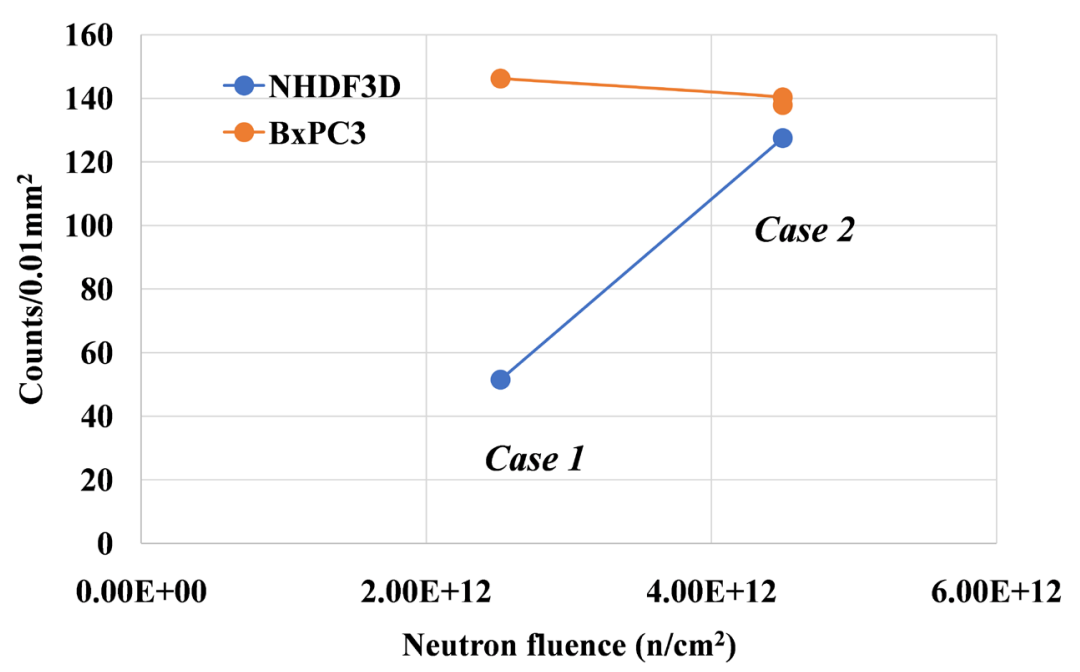

Figure 6. The relationship between irradiation dose and track density. The increase rate of NHDF3D and BxPC3 is different with the increase of the irradiation amount, and the increase rate of the track density of NHDF3D is remarkable. On the other hand, it can be seen that the increase rate of $\mathrm{BxPC} 3$ is almost saturated, however, in Case 2, the rate of increase is similar to that of normal cells.

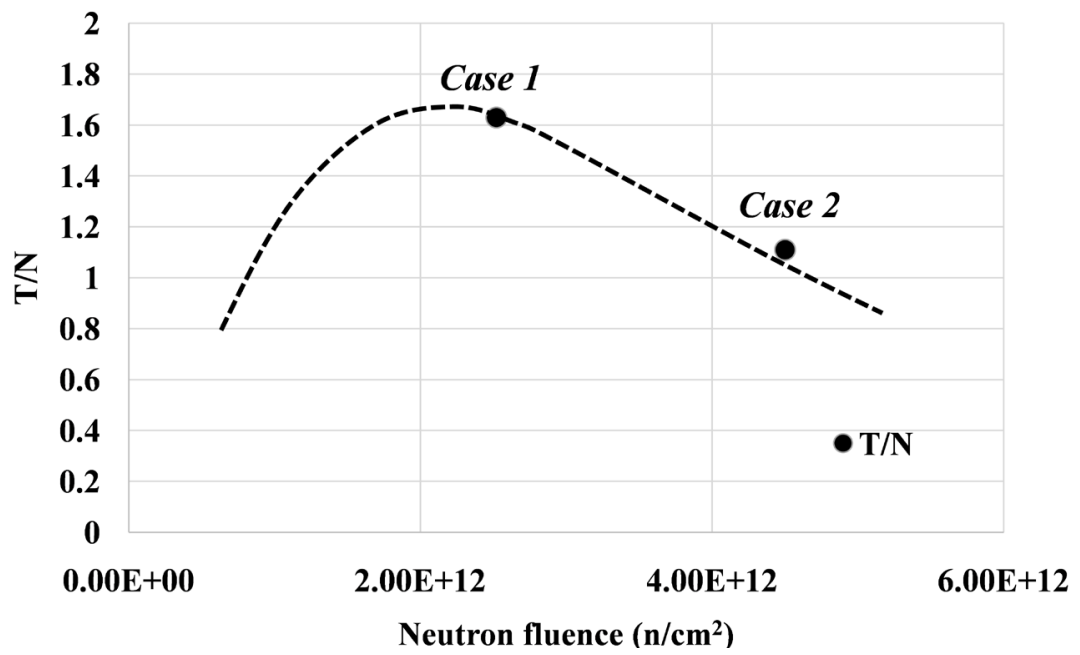

Figure 7. The relationship between irradiation dose and $T / N$ ratio. The $T / N$ ratio increases as the neutron irradiation dose increases, it turns to decrease when the irradiation dose in Case 1 is exceeded. In other words, in Case 2 from the viewpoint of BNCT treatment, radiation damage to the normal tissue becomes significant and there is a possibility of over-irradiation.

In conclusion, our in vitro model of tumor tissue for BNCT demonstrated the pharmacokinetics of BPA and the efficacy of neutron irradiation by direct observation of $\alpha$-ray/recoiled Li particle tracks that are corresponding to the distribution of BxPC3 cells [1]. Furthermore, in this paper, following the previous paper, heavy irradiation was performed using the same artificial tumor tissue. As a result, it became clear that selection of the optimal dose, which is one of the most important treatment conditions for BNCT treatment, is possible to some extent by an evaluation method using an artificial tumor tissue. 


\section{Conflicts of Interest}

The authors declare no conflicts of interest regarding the publication of this paper.

\section{References}

[1] Ishiyama, Asano, Y., Suzuki, M., et al. (2019) In Vitro Evaluation System of Pharmacokinetics and Irradiation Effect in Boron Neutron Capture Therapy (BNCT) Using Three-Dimentional Artificial Human Tumor Tissue Model. Journal of Cancer Therapy, 10, 835-845. https://doi.org/10.4236/jct.2019.1010071

[2] Masunaga, S., Sakurai, Y., Tanaka, H., et al. (2014) The Dependency of Compound Biological Effectiveness Factors on the Type and Concentration of Administered Neutron Capture Agents in Boron Neutron Capture Therapy. Springerplus, 3, 128. https://doi.org/10.1186/2193-1801-3-128

[3] Ishiyama, S., Baba, Y., Fujii, R., Nakamura, M. and Imahori, Y. (2012) Synthesis of Lithium Nitride for Neutron Production Target of BNCT by In-Situ Lithium Deposition and Ion Implantation. Nuclear Instruments and Methods in Physics Research, 293, 42-47. https://doi.org/10.1016/j.nimb.2012.09.016

[4] Ishiyama, S., Baba, Y., Fujii, R., Nakamura, M. and Imahori, Y. (2013) Thermal Stability of BNC Neutron Production Target Lithium Synthesized by In-Situ Lithium Deposition and Ion Implantation. Materials Transactions, 54, 1760-1764. https://doi.org/10.2320/matertrans.M2013062

[5] Ishiyama, S. and Imahori, Y. (2014) Deterministic Parsing Model of CBE Factor for Intra-Organ ${ }^{10} \mathrm{~B}$ Distribution in Boron Neutron Capture Therapy. Journal of Cancer Therapy, 5, 1388-1398. https://doi.org/10.4236/jct.2014.514140

[6] Ishiyama, S., Imahori, Y., Itami, J. and Hanna, V. (2015) Determination of the Compound Biological Effectiveness (CBE) Factors Based on the Ishiyama-Imahori Deterministic Parsing Model with the Dynamic PET Technique. Journal of Cancer Therapy, 6, 759-766. https://doi.org/10.4236/jct.2015.68083

[7] Suzuki, M., et al. (2014) Boron Neutron Capture Therapy Outcomes for Advanced or Recurrent Head and Neck Cancer. Journal of Radiation Research, 55, 146-153. https://doi.org/10.1093/jrr/rrt098

[8] Suzuki, M., et al. (2007) First Attempt of Boron Neutron Capture Therapy (BNCT) for Hepatocellular Carcinoma. Japanese Journal of Clinical Oncology, 37, 376-381. https://doi.org/10.1093/jico/hym039

[9] Ichihashi, M., et al. (1982) Specific Killing Effect of 10B1-Para-boronophenylalanine in Thermal Neutron Capture Therapy of Malignant Melanoma, in Vitro Radiobiological Evaluation. Journal of Investigative Dermatology, 78, 215-218. https://doi.org/10.1111/1523-1747.ep12506489

[10] Hiratsuka, J., et al. (1982) RBEs of Thermal Neutron Capture Therapy and 10B(n, alpha)7Li Reaction on Melanoma Bearing Hamsters. Pigment Cell Research, 2, 352-355. https://doi.org/10.1111/j.1600-0749.1989.tb00219.x

[11] Matsusaki, M. (2012) Development of Three-Dimensional Tissue Models Based on Hierarchical Cell Manipulation Using Nanofilms. Bulletin of the Chemical Society of Japan, 85, 401-414. https://doi.org/10.1246/bcsj.20110194

[12] Matsusaki, M., Case, C.P. and Akashi, M. (2014) Three-Dimensional Cell Culture Technique and Pathophysiology. Advanced Drug Delivery Reviews, 74, 95-103. https://doi.org/10.1016/j.addr.2014.01.003 
[13] Asano, Y., Odagiri, T., Oikiri, H., Matsusaki, M., Alashi, M. and Shimoda, H. (2017) Construction of Artificial Human Peritoneal Tissue by Cell-Accumulation Technique and Its Application for Visualizing Morphological Dynamics of Cancer Peritoneal Metastasis. Biochemical and Biophysical Research Communications, 494, 213-219. https://doi.org/10.1016/j.bbrc.2017.10.050

[14] Nishiguchi, A., Matsusaki, M., Kano, M.R., Nishihara, H., Okano, D., Asano, Y., Shimoda, H., Kishimoto, S., Iwai, S. and Akashi, M. (2018) In Vitro 3D Blood/ Lymph-Vascularized Human Stromal Tissues for Preclinical Assays of Cancer Metastasis. Biomaterials, 179, 144-155.

https://doi.org/10.1016/j.biomaterials.2018.06.019

[15] Tan, M.H., Nowak, N.J. and Loor, R. (1986) Characterization of a New Primary Human Pancreatic Tumor Line. Cancer Investigation, 4, 15-23. https://doi.org/10.3109/07357908609039823

[16] Deer, E.L., Hernandes, J.G., Coursen, J.D., Shear, J.E., et al. (2010) Phenotype and Genotype of Pancreatic Cancer Cell Lines. Pancreas, 39, 425-435.

https://doi.org/10.1097/MPA.0b013e3181c15963 